\title{
INTEGRAL CLOSURES OF UNCOUNTABLE COMMUTATIVE REGULAR RINGS
}

\author{
L. LIPSHITZ ${ }^{1}$
}

\begin{abstract}
Necessary and sufficient conditions are given for a commutative
\end{abstract} regular ring to have a prime integrally closed extension.

In this paper we give necessary and sufficient conditions for a commutative regular ring $R$ to have a prime integral closure. $\ln [1]$ it was shown that for a commutative regular ring $R$ to have a prime integral closure, it is necessary that every polynomial $p(x)$ in $R[x]$ have an unambiguous factor (see definitions below), and that in the case that $R$ is countable this condition is also sufficient. An example was given to show that this condition is not sufficient if $R$ is uncountable. It was also seen in [1] that if $R$ has a prime integral closure, then this closure is unique. I would like to thank Bonnie Gold and Gadi Moran for helpful conversations during the preparation of this paper.

Definitions. (1) $K_{C R}$ is the theory of commutative regular rings;

$$
K_{\overline{C R}}=K_{C R} \cup\{\text { every monic polynomial has a root }\}
$$

is the theory of integrally closed commutative regular rings.

(2) If $R \vDash K_{C R}$ and $p(x) \in R[x]$, we call $p(x)$ unambiguous if on no nonzero idempotent $e$ is it the case that $p(x)=u(x) v(x)$ with $(u(x), v(x))=1$ on $e$. (An identity holds on $e$ if it holds in $R e$.) This condition is equivalent to $p(x)$ being a power of an irreducible polynomial at every point of $S_{R}$, the Stone space of $R(=\operatorname{Spec}(R))$.

$$
T=K_{C R} \cup\{\text { every polynomial has an unambiguous factor }\} .
$$

(3) If $R \vDash K_{C R}$ and $R \subset \bar{R} \vDash K_{\overline{C R}}$, we call $\bar{R}$ a prime extension of $R$ to a model of $K_{\overline{C R}}$, or an (in fact the) integral closure of $R$ if whenever $f: R \rightarrow R_{1} \vDash K_{\overline{C R}}$ is an embedding, $f$ extends to an embedding of $\bar{R}$ into $R_{1}$. If we drop the condition that $\bar{R} \vDash K_{\overline{C R}}$ we call $\bar{R}$ a prime extension of $R$.

(4) If $R \vDash K_{C R}$ and $R \subset \bar{R} \vDash K_{C R}$, we call $\bar{R}$ sequentially prime over $R$ if $\bar{R}=\cup_{\alpha<\lambda} \bar{R}_{\alpha}$ with $R_{0}=R, R_{\delta}=\cup_{\alpha<\delta} R_{\alpha}$ for limit ordinals $\delta<\lambda$ and $R_{\alpha+1}=R_{\alpha}\left[a_{\alpha}\right]$, with $a_{\alpha}$ a root of an unambiguous polynomial $p_{\alpha}(x) \in R_{\alpha}[x]$. (In other words, $\bar{R}$ can be realized as a sequence of one element extensions, each prime over the previous ones-see [1].)

Received by the editors February 28, 1975 and, in revised form, June 21, 1975.

AMS (MOS) subject classifications (1970). Primary 13L05, 13B20; Secondary 02H15.

Key words and phrases. Commutative regular rings, integrally closed rings, prime model extensions.

1 This research supported by NSF grant GP43749. 
(5) Let $R \vDash K_{C R}$. We call $R$ thin if there is a set $\mathscr{P} \subset R_{i}[x]$, where $R_{i}$ is the inseparable closure of $R$-see [1], such that (a) every polynomial $p(x) \in \mathscr{P}$ is normal (in the sense that adjoining one root of $p(x)$ splits $p(x)$ into linear factors) and unambiguous. (b) If $R^{\prime} \supset R$ splits every polynomial in $\mathscr{P}$ and is generated over $R$ by the roots of these polynomials, then $R^{\prime} \vDash K_{\overline{C R}}$. (c) Each $p(x) \in \mathscr{P}$ is defined and monic on some idempotent $e_{p}(\varepsilon R)$ and $p(x)=p(x) e_{p}$. (d) If $A \subset \mathscr{P}$ is countable, there is a countable $B$ with $A \subset B \subset \mathscr{P}$ such that if $R^{\prime}$ results from $R$ by adjoining roots of all the polynomials $p(x) \in B$ (in the sequentially prime way-see [1]), then in $R^{\prime}[x]$ every polynomial $p(x) \in \mathscr{P}$ factors on $e_{p}$ into unambiguous monic factors. We shall call such a $\mathscr{P}$ a thin basis for $R$.

We shall show that if $\bar{R}$ is prime over $R$, then $\bar{R}$ is sequentially prime over $R$ and consequently that $R$ has a prime integral closure if and only if $R$ is thin.

REMARK. In definition (5) above the only important conditions are (b) and (d); i.e. if we have a set of polynomials which satisfies (b) and (d), then we can construct a set satsifying (a)-(d). Notice also that if $R$ is thin,then $R \vDash T$.

From now on, when $R \vDash T$, we shall assume that $R$ is inseparably closed (i.e. every purely inseparable polynomial in $R[x]$ has a root). This involves no loss of generality since the inseparable closure $R_{i}$ of $R$ always exists and is prime and in fact sequentially prime over $R$. If $R$ is inseparably closed instead of unambiguous polynomials, we can talk of irreducible polynomials (see [1]). Also all irreducible polynomials are then separable and, consequently, we have the primitive element theorem holding.

Let $R \vDash T$ and let $\mathscr{P}=\{p(x) \in R[x] \mid p(x)$ is normal, monic and unambiguous\}. Let

$$
R^{*}=\prod_{j \in J} R\left[\left\{x_{p} \mid p \in \mathscr{P}\right\}\right]_{j}
$$

where the product is over all isomorphism types of $R\left[\left\{x_{p} \mid p \in \mathcal{P}\right\}\right]$ such that $p\left(x_{p}\right)=0$ for all $p \in \mathcal{P}$. Let $\tilde{R}$ be the subring of $R^{*}$ generated by the sequences $x_{p}=\left\{x_{p, j}\right\}_{j \in J}$, over $R$. It follows from Lemma 1 of [2] or Lemma 2 of [1] that $\tilde{R}$ is a commutative regular ring. It is not hard to see that $\tilde{R} \vDash K_{\overline{C R}}\left(\tilde{R}\right.$ is algebraically closed at each point of $S_{\tilde{R}}=\operatorname{Spec}(\tilde{R})$ and since $S_{\tilde{R}}$ is compact, $\tilde{R}$ is integrally closed). $\tilde{R}$ is a free closure of $R$ in the sense that if $R \subset R_{1} \vDash K_{\overline{C R}}$, then there is a homomorphism of $\tilde{R}$ into $R_{1}$ over $R$-in fact one of the projections will do.

Suppose that $R$ has a prime integral closure $\bar{R}$. Let $\nu: \bar{R} \rightarrow \tilde{R}$ be a fixed embedding over $R$. For each $\beta \in \bar{R}$ there is a finite set $X_{\beta} \subset\left\{x_{p} \mid p \in \mathscr{P}\right\}$ $\subset \tilde{R}$ such that $\nu(\beta) \in R\left[x_{p} \mid x_{p} \in X_{\beta}\right]$. If $A \subset \bar{R}$, define $A^{\prime} \subset \bar{R}$ as follows: $A_{0}=A, A_{i+1}=\left\{\right.$ all roots in $R$ of polynomials $p(x) \in R[x]$ such that $x_{p}$ $\left.\in \cup_{\beta \in A_{i}} X_{\beta}\right\}$ and $A^{\prime}=\cup_{i \in \omega} A_{i}$. Notice that if $p(x) \in R[x]$, then all the roots of $p(x)$ in $R$ are generated by a finite number of roots over $R$, since $S_{\bar{R}}=S_{R}$. It follows that if $\overline{\bar{A}} \leqslant \aleph_{0}$, then $R\left[A^{\prime}\right]$ is countably generated over $R$ and, in fact, if $A \subset B$ with $B-A$ countable, then $R\left[B^{\prime}\right]$ is countably generated over $R\left[A^{\prime}\right]$.

Let $\bar{R}=R\left[\left\{x_{\alpha} \mid \alpha<\lambda\right\}\right]$ where each $x_{\alpha}$ is a root of a polynomial $p(x) \in$ op. Define $A_{\alpha}=\left\{x_{\gamma} \mid \gamma<\alpha\right\}$. $\bar{R}_{\alpha}=R\left[A_{\alpha}^{\prime}\right] \subset \bar{R}$ and $\tilde{R}_{\alpha}=R\left[\left\{x_{p} \in \tilde{R} \mid a\right.\right.$ is a root of $p(x)$ for some $\left.\left.a \in \bar{R}_{\alpha}\right\}\right] \subset \tilde{R}$. 
It is clear that $\bar{R}_{\alpha}=\nu^{-1}\left(\tilde{R}_{\alpha}\right)$, that $\bar{R}_{\delta}=\cup_{\alpha<\delta} \bar{R}_{\alpha}$ for limit ordinals $\delta \leqslant \lambda$, that $\bar{R}_{\lambda}=\bar{R}$ and that $\bar{R}_{\alpha+1}$ is countably generated over $\bar{R}_{\alpha}$.

LeMma 1. (i) $\bar{R}_{\alpha}$ is prime over $R$.

(ii) $\bar{R}_{\alpha+1}$ is prime over $\bar{R}_{\alpha}$.

(iii) $\bar{R}_{\alpha+1}$ is sequentially prime over $\bar{R}_{\alpha}$.

Proof. (i) is trivial.

(ii) Since $\tilde{R}_{\alpha}$ is free over $R$ there is a projection $\mu: \tilde{R}_{\alpha} \rightarrow \bar{R}_{\alpha}$ over $R$. It is easy to see that $\mu \circ \nu$ is an automorphism of $\bar{R}_{\alpha}$. Let $g^{\prime}=\operatorname{Ker}(\mu) \subset \tilde{R}_{\alpha}$ and let $g=g^{\prime} \tilde{R}$. Then since $\tilde{R}$ and $\tilde{R}_{\alpha}$ are models of $K_{C R}, g \cap \tilde{R}_{\alpha}=g^{\prime}$. Also $\mu: \tilde{R}_{\alpha} / \mathcal{G}^{\prime} \rightarrow \bar{R}_{\alpha}$ is an isomorphism. It is easy to see that $\tilde{R} / \mathscr{G}$ is free over $\bar{R}_{\alpha}=\tilde{R}_{\alpha} / \mathscr{G}^{\prime}$ (in the same sense that $\tilde{R}$ is free over $R$ ). Let $\bar{R}_{\alpha} \subset R_{2} \vDash K_{\overline{C R}}$. Then there is a homomorphism $\mu_{1}: \tilde{R} / \mathscr{G} \rightarrow R_{2}$ over $\bar{R}_{\alpha}$ so that $\mu_{1} \circ \nu: \bar{R} \rightarrow R_{2}$ is an embedding. Hence $\bar{R}$ (and thus $\bar{R}_{\alpha+1}$ ) is prime over $R_{\alpha}$. Hence, since $\bar{R}_{\alpha+1}$ is countably generated over $R_{\alpha}$, by the remark following Theorem 2 of [1], $\bar{R}_{\alpha+1}$ is sequentially prime over $\bar{R}_{\alpha}$, and (ii) and (iii) are proved.

Corollary. If $\bar{R}$ is a prime integral closure of $R\left(\vDash K_{C R}\right)$, then $\bar{R}$ is sequentially prime over $R$.

Proof. The results of [1] show that $\bar{R} \vDash T$. The inseparable closure $R_{i}$ of $R$ is always sequentially prime over $R$ and $R_{i} \vDash T$ so the above construction and Lemma 1 show that $\bar{R}$ is sequentially prime over $R_{i}$.

Lemma 2. If $\bar{R}$ is the prime integral closure of $R$, then $R$ is thin.

Proof. Let $\bar{R}=\cup_{\alpha<\lambda} R_{\alpha}$ where $\bar{R}_{\alpha+1}=\bar{R}_{\alpha}\left[a_{\alpha}\right]$ and $p_{\alpha}\left(a_{\alpha}\right)=0$ with $p_{\alpha}(x) \in \bar{R}_{\alpha}[x]$ irreducible. Let $p_{\alpha}^{*}(x) \in R[x]$ be the unique irreducible polynomial in $R[x]$ such that $p_{\alpha}(x) \mid p_{\alpha}^{*}(x)$. Without loss of generality we may assume that $p_{\alpha}(x)$ and $p_{\alpha}^{*}(x)$ are normal (see the proof of Lemma 1). A set $A \subset \bar{R}-R$ is called downwardly closed if: (i) if $a \in R[A]$ and at some point $z \in S_{R}$ the first time $a(z)$ occurs in the sequence $\bar{R}_{\gamma} / z$ is at stage $\alpha$ with $a(z)$ being a combination over $R$ of $a_{\gamma_{1}}, \ldots, a_{\gamma_{n}}$ say, then $a_{\gamma_{i}} \in R[A]$ for $i=1$, $\ldots, n$; and (ii) $A=A^{\prime}$. In the proof of Lemma 1 we saw that if $A=A^{\prime}$, then $R[A] \vDash T$, so if $A$ is downwardly closed then $R[A] \vDash T$. For each downwardly closed $A \subset \bar{R}$ let $Y_{A}^{\alpha}$ be a factoring of $p_{\alpha}^{*}$ into irreducible factors in $R[A]$. Call two such factorings $Y_{A_{1}}^{\alpha}$ and $Y_{A_{2}}^{\alpha}$ essentially different if at some point $z \in S_{R}$ they are different. We now claim that for fixed $\alpha$ there are only finitely many essentially different $Y_{A}^{\alpha}$,s (with $A$ downwardly closed). This follows from the fact that $p_{\alpha}^{*}$, factors only finitely often in the well-ordered sequence $\bar{R}_{\gamma}$ since each $\bar{R}_{\gamma} \vDash T$, and that $S_{R}$ is compact. We leave the details to the reader. For each $\alpha$ choose idempotents $e_{\alpha, i}, i=1, \ldots, n_{\alpha}$ such that for any downwardly closed $A$ each $p_{\alpha}^{*}(x) e_{\alpha, i}$ factors into monic irreducible factors on $e_{\alpha, i}$ for each $i$. Let

$$
\mathcal{P}=\left\{p_{\alpha}^{*}(x) e_{\alpha, i} \mid \alpha<\lambda, i=1, \ldots, n_{\alpha}\right\} .
$$

Certainly $\mathscr{P}$ satisfies all the conditions of definition (5) except perhaps (d). Let $A \subset \mathcal{P}$ with $\bar{A}=\aleph_{0}$ and let $B_{1}$ be the downward closure of $A$ (defined as follows: For each $a \in R[A]-R$ and each $z \in S_{R}$, adjoin to $A$ the elements 
$a_{\gamma_{1}}, \ldots, a_{\gamma_{n}}$ defined above. Since $S_{R}$ is compact, this will only involve considering a finite number of $z$ 's. Call the new set $D$. Let $A_{1}=D^{\prime}$. Obtain $A_{i+1}$ from $A_{i}$ in the same way that $A_{1}$ was obtained from $A$. The downward closure of $A$ is $\cup_{i<\omega} A_{i}$ ). We must show that there is a countable subset $B \subset B_{1}$ such that adjoining roots for all polynomials in $B$ (in the prime way) causes every polynomial in $B_{1}$ to split. Since $\bar{R}$ is separable over $R$ for each $\left\{a_{1}, \ldots, a_{n}\right\} \subset \bar{R}$ there are essentially only finitely many regular rings between $R$ and $R\left[a_{1}, \ldots, a_{n}\right]$. By this we mean that there is a finite set of regular rings $R_{j}, j=1, \ldots, k$, with $R_{j} \subset R\left[a_{1}, \ldots, a_{n}\right]$ and $R_{j}$ finitely generated over $R$ such that at each point $z \in S_{R}$, if $R_{z}$ denotes the field (i.e. stalk) above $z$, then all the fields between $R_{z}$ and $R\left[a_{1}, \ldots, a_{n}\right]_{z}$ occur among the $R_{j z}$. For each $\left\{a_{1}, \ldots, a_{n}\right\} \subset A$ we can look at the rings $R_{j}$ defined as above and choose a finite set of generators $A_{j}$ for $R_{j}$ over $R$. Let $A$ be the union of all these $A_{j}$ for all finite subsets $\left\{a_{1}, \ldots, a_{n}\right\}$ of $A$. Then $\bar{A}$ is countable and in obtaining $D$ from $A$ as above instead of considering all elements of $R[A]-R$ we need only consider all elements of $\bar{A}$. Call this set $\bar{D}$. Let $A_{1}=\bar{D}^{\prime}$ etc. and $B=\cup_{i<\omega} A_{i}$. Then $B$ is countable and downwardly closed. In fact $R[B]$ $=R$ [downward closure of $A$ ]. From the definition of $\mathcal{P}$ it is clear that $B$ has the required properties.

Lemma 3. If $R \subset R_{1} \subset R_{2}$ with $R_{1} \vDash T$ and $R_{j}(j=1,2)$ prime over $R$ then $R_{2}$ is prime over $R_{1}$.

Proof. Let $\tilde{R}_{1}$ be constructed from $R_{1}$ as above. We then have

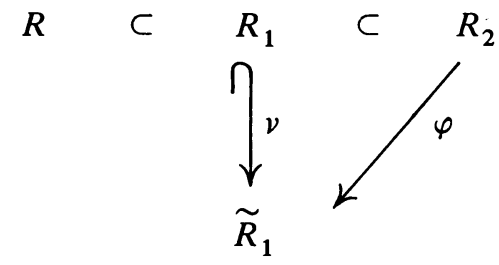

Proof. Let $\mathcal{P}$ be a thin basis for $R$. Let $A \subset \mathcal{P}$. Then there is a $B$ $(A \subset B \subset \mathcal{P})$ with $\overline{\bar{A}}+\aleph_{0}=\overline{\bar{B}}+\aleph_{0}$ so that every $p \in \mathcal{P}$ factors in $R_{B}$ (obtained by adjoining roots of polynomials in $B$ ) into the product of irreducible monic factors on $e_{p}$.

We prove by induction on $\overline{\bar{A}}$ that if $A \subset \mathscr{P}$, then there is a sequentially prime extension $R_{A}$ of $R$ which splits every polynomial in $A$ and with $R_{A} \vDash T$, and such that in $R_{A}[x]$ every polynomial $p \in \mathscr{P}$ factors into the product of monic irreducible factors on $e_{p}$. If $A$ is countable this is trivial. Suppose the assertion is true for all cardinals $<\overline{\bar{A}}$. Let $B$ correspond to $A$ as above. Write $A=\cup_{\alpha}<\lambda A_{\alpha}$ with $A_{\delta}=\cup_{\alpha<\delta} A_{\alpha}$ for limit ordinals $\delta \leqslant \lambda, A_{\alpha+1} \supset A_{\alpha}$ and $\overline{\bar{A}} \bar{A}_{\alpha}<\overline{\bar{A}}$ for all $\alpha<\lambda$. Let $B=\cup_{\alpha<\lambda} B_{\alpha}$ with $B_{\alpha}$ corresponding to $A_{\alpha}$ as above. Then by induction $R_{B_{\alpha}}$ exists for each $\alpha<\lambda$. It is clear that $R_{B_{\alpha}} F T$ (since in $R_{B_{\alpha}}$ every polynomial in $\mathcal{P}$ factors into a product of monic irreducible factors) for each $\alpha<\lambda$. Thus by Lemma $3, R_{B_{\alpha+1}}$ is prime over $R_{B_{\alpha}}$ and hence $R_{B}=\cup_{\alpha<\lambda} R_{B_{\alpha}}$ is prime over $R$.

From Corollary 1 and Lemma 4 we immediately get the

THEOREM. If $R \vDash K_{C R}$ then $R$ has a prime integral closure if and only if $R$ is thin. 
where $\varphi$ is an embedding of $R_{2}$ into $\tilde{R}_{1}$ over $R$ which exists because $R_{2}$ is prime over $R$. This diagram need not commute, but we do have $\nu(r)=\varphi(r)$ for $r \in R$. We shall show that there exists an automorphism $\psi$ of $\tilde{R}_{1}$ over $R$ such that the above diagram with $\phi$ replaced by $\psi^{-1} \circ \varphi$ does commute. The lemma then follows from the freeness properties of $\tilde{R}_{1}$ over $R_{1}$.

$\tilde{R}_{1}$ is generated by the $x_{p}, p \in \mathcal{P}$, over $R_{1}$. For $a \in R_{1}$ let $a_{i}, i=1, \ldots, n_{a}$, denote the conjugates of $a$ over $R$, and for $p(x) \in \mathscr{P}$ let $p_{i}(x), i=1, \ldots, n_{p}$, denote the conjugates of $p(x)$ over $R$. Notice that if $p(x) \in \mathscr{P}$, then $p_{i}(x) \in \mathscr{P}$, and since $R_{1} \vDash T,\left(p_{i}(x), p_{j}(x)\right)=1$ for $i \neq j$.

For $a \in R_{1}$ we have $\varphi(a)=\sum a_{i} e_{i}$ where the $e_{i}$ are disjoint idempotents of $\tilde{R}_{1}$ and $\sum e_{i}=1$. Similarly we have $\varphi(p(x))=\sum_{i=1}^{n_{p}} p_{i}(x) e_{i}$.

Define $\psi: \tilde{R}_{1} \rightarrow \tilde{R}_{1}$ as follows:

$$
\begin{aligned}
\psi(a) & =\varphi \circ \nu^{-1}(a) \text { for } a \in \nu\left(R_{1}\right), \\
\psi\left(x_{p}\right) & =\sum_{i=1}^{n_{p}} x_{p_{i}} e_{i} .
\end{aligned}
$$

It is obvious that $\psi$ is a homomorphism because of the freeness properties of $\tilde{R}_{1}$ over $R_{1} . \psi$ is locally one-to-one (i.e. on each stalk) and hence one-to-one. Also $x_{p} \in$ Range $(\psi)$ so $\psi$ is onto. Therefore $\psi$ is an automorphism with the required properties.

LEMMA 4. If $R$ is thin, then $R$ has a prime integral closure.

Remark. The condition that $R$ be thin is not a first order condition since every countable model of $T$ is thin. Hence for $R$ uncountable the necessary and sufficient condition for $R$ to have a prime integral closure is not first order, while for countable $R$ it is.

\section{REFERENCES}

1. L. Lipshitz, Commutative regular rings with integral closure, Trans. Amer. Math. Soc. 211 (1975), 161-170.

2. - The real closure of a commutative regular f-ring Fund. Math. (to appear).

Department of Mathematics, Purdue University, West Lafayette, Indiana 47907 